\title{
CONSTRUCTION OF LAGRANGIAN SURFACES IN COMPLEX EUCLIDEAN PLANE WITH LEGENDRE CURVES
}

\author{
BANG-YEN CHEN
}

\begin{abstract}
An important problem in the theory of Lagrangian submanifolds is to find nontrivial examples of Lagrangian submanifolds in complex Euclidean spaces with some given special geometric properties. In this article, we provide a new method to construct Lagrangian surfaces in the complex Euclidean plane $\mathbf{C}^{2}$ by using Legendre curves in $S^{3}(1) \subset \mathbf{C}^{2}$. We also investigate intrinsic and extrinsic geometric properties of the Lagrangian surfaces in $\mathbf{C}^{2}$ obtained by applying our construction method. As an application we provide some new families of Hamiltonian minimal Lagrangian surfaces in $\mathbf{C}^{2}$ via our construction method.
\end{abstract}

\section{Introduction}

An immersion $f: M^{n} \rightarrow \tilde{M}^{n}$ of an $n$-manifold $M^{n}$ into a Kaehler $n$ manifold $\tilde{M}^{n}$ is called a Lagrangian immersion if the complex structure $J$ of $\tilde{M}^{n}$ interchanges each tangent space of $M^{n}$ with its corresponding normal space. Lagrangian submanifolds appear naturally in the context of classical mechanics and mathematical physics. For instance, the systems of partial differential equations of Hamilton-Jacobi type lead to the study of Lagrangian submanifolds and foliations in the cotangent bundle. Furthermore, Lagrangian submanifolds are part of a growing list of mathematically rich special geometries that occur naturally in string theory.

An important problem in the theory of Lagrangian submanifolds is to find non-trivial examples of Lagrangian submanifolds with some given special geometric properties. For instance, a method was given in [23] to construct an important family of special Lagrangian submanifolds in $\mathbf{C}^{n}$ with large symmetric groups. Moreover, a method was introduced in [17] to construct Lagrangian submanifolds of constant sectional curvature by utilizing twisted products decompositions of real space forms. Furthermore, R. Aiyama introduced in [1, 2] a spinor-like representation formula which parameterizes immersions through

2000 Mathematics Subject Classification. Primary 53C40, 53D12; Secondary 53C42.

Key words and phrases. Lagrangian immersion, Legendre curve, Lagrangian surface of constant curvature, Hamiltonian minimal Lagrangian surfaces, Lagrangian catenoid.

Received May 26, 2005; revised August 23, 2005. 
two complex functions $F_{1}, F_{2}$ and a real one (the Lagrangian angle $\beta$ ). Aiyama's formula is very useful to construct many examples of Lagrangian surfaces in $\mathbf{C}^{2}$.

For a Lagrangian submanifold in a Kaehler manifold with mean curvature vector $H$ and shape operator $A$, the dual 1 -form $\alpha_{H}$ of $J H$ is the well-known Maslov form (up to constants). A non-minimal Lagrangian submanifold is called Maslovian if its Maslov vector field $J H$ is a principal direction of $A_{H}$ (see $[12,18])$. Maslovian Lagrangian surfaces of constant curvature in $\mathbf{C}^{2}$ have been classified in $[10,11]$.

A regular curve $z: I \rightarrow S^{3}(r) \subset \mathbf{C}^{2}$ in the hypersphere $S^{3}(r)$ of radius $r$ centered at the origin of $\mathbf{C}^{2}$ is called a Legendre curve if $\left\langle z^{\prime}(t), i z(t)\right\rangle=0$ holds identically.

In [7], the author introduced the notion of complex extensors to construct a special family of Maslovian Lagrangian submanifolds in $\mathbf{C}^{n}$. Furthermore, he also established a method in [9] to construct flat Lagrangian submanifolds in $\mathbf{C}^{n}$ by using special Legendre curves in $S^{2 n-1}(1) \subset \mathbf{C}^{n}$. In particular, his result implies that, for any unit speed Legendre curve $z: I \rightarrow S^{3}(1) \subset \mathbf{C}^{2}$ and any realvalued function $p(t)$ defined on an open interval $I$ containing 0 , the map

$$
L(s, t)=s z(t)+\int_{0}^{t} p(t) z^{\prime}(t) d t
$$

defines a flat Maslovian Lagrangian surface in $\mathbf{C}^{2}$.

In this article, we extends the idea of [9] to provide a more general method than [9] to construct Lagrangian surfaces in $\mathbf{C}^{2}$ by using Legendre curves in $S^{3}(1) \subset \mathbf{C}^{2}$. In this article, we also investigate extrinsic geometric properties of Lagrangian surfaces in $\mathbf{C}^{2}$ obtained in such way. Moreover, we completely classify minimal Lagrangian surfaces as well as Lagrangian surfaces of constant curvature in $\mathbf{C}^{2}$ which are obtained by applying our construction method. As an application we apply our construction method to provide some new families of Hamiltonian minimal Lagrangian surfaces in $\mathbf{C}^{2}$.

\section{Preliminaries}

Let $f: M \rightarrow \mathbf{C}^{2}$ be an isometric immersion of a surface $M$ into the complex Euclidean plane $\mathbf{C}^{2}$. We denote the Riemannian connections of $M$ and $\mathbf{C}^{2}$ by $\nabla$ and $\tilde{\nabla}$, respectively; and by $D$ the connection on the normal bundle of the surface.

The formulas of Gauss and Weingarten are given respectively by

$$
\begin{aligned}
\tilde{\nabla}_{X} Y & =\nabla_{X} Y+h(X, Y), \\
\tilde{\nabla}_{X} \xi & =-A_{\xi} X+D_{X} \xi
\end{aligned}
$$

for tangent vector fields $X$ and $Y$ and normal vector field $\xi$. The second fundamental form $h$ is related to the shape operator $A_{\xi}$ by 


$$
\langle h(X, Y), \xi\rangle=\left\langle A_{\xi} X, Y\right\rangle .
$$

The mean curvature vector $H$ of $M$ in $\mathbf{C}^{2}$ is defined by $H=\frac{1}{2}$ trace $h$. The length of $H$ is called the mean curvature function.

If we denote the Riemann curvature tensor of $\nabla$ by $R$, then the equations of Gauss and Codazzi are given respectively by

$$
\begin{aligned}
\langle R(X, Y) Z, W\rangle & =\langle h(X, W), h(Y, Z)\rangle-\langle h(X, Z), h(Y, W)\rangle, \\
(\nabla h)(X, Y, Z) & =(\nabla h)(Y, X, Z),
\end{aligned}
$$

where $X, Y, Z, W$ are vector fields tangent to $M$ and $\nabla h$ is defined by

$$
(\nabla h)(X, Y, Z)=D_{X} h(Y, Z)-h\left(\nabla_{X} Y, Z\right)-h\left(Y, \nabla_{X} Z\right) .
$$

When $M$ is a Lagrangian surface in $\mathbf{C}^{2}$, we also have (cf. [20])

$$
\begin{aligned}
D_{X} J Y & =J \nabla_{X} Y, \\
\langle h(X, Y), J Z\rangle & =\langle h(Y, Z), J X\rangle=\langle h(Z, X), J Y\rangle .
\end{aligned}
$$

The following lemma was obtained in [8].

LEMMA 2.1. We have the following:

(a) If $z: I \rightarrow S^{3}(1) \subset \mathbf{C}^{2}$ is a unit speed curve satisfying

$$
z^{\prime \prime}(t)-i \lambda(t) z^{\prime}(t)+z(t)=0
$$

for some nonzero real-valued function $\lambda$, then $z=z(t)$ is a Legendre curve.

(b) Conversely, if $z: I \rightarrow S^{3}(1) \subset \mathbf{C}^{2}$ is a unit speed Legendre curve, then it satisfies (2.4) with $\lambda$ equal to the curvature function of the Legendre curve in $S^{3}(1)$.

Remark 2.1. A unit speed curve $z: I \rightarrow S^{3}(r) \subset \mathbf{C}^{2}$ satisfying (2.4) with $\lambda=0$ is a great circle. A great circle in $S^{3}(r)$ can be either Legendre or nonLegendre. For examples, $z(t)=(\cos t, \sin t)$ is a Legendre curve in $S^{3}(1)$ and $z(t)=\left(e^{i t}, 0\right)$ in $S^{3}(1)$ is not a Legendre curve.

We also need the following lemma.

Lemma 2.2. Let $A, B$ be two vectors in $\mathbf{C}^{n}$ and $z, w$ be two complex numbers. Then we have

$$
\begin{aligned}
\langle z A, w B\rangle & =\langle z, w\rangle\langle A, B\rangle+\langle i z, w\rangle\langle A, i B\rangle, \\
\langle z A, i w B\rangle & =\langle z, w\rangle\langle A, i B\rangle+\langle z, i w\rangle\langle A, B\rangle,
\end{aligned}
$$

where $\langle z, w\rangle=\operatorname{Real}(z \bar{w})$ denotes the real part of the complex number $z \bar{w}, \bar{w}$ the complex conjugate of $w$, and $\langle A, B\rangle$ denotes the canonical inner product of the vectors $A$ and $B$ in the complex Euclidean n-plane $\mathbf{C}^{n}$.

Proof. Follows easily by straightforward computation. 


\section{Interaction of Legendre curves and Lagrangian surfaces}

For each $w \in \mathbf{C}^{n}$ we put $|w|=\sqrt{\langle w, w\rangle}$. We also put $\mathbf{C}^{*}=\mathbf{C}-\{0\}$. Recall that a curve is called regular if its speed function is nowhere zero.

The following result provides a simple method to construct many examples of Lagrangian surfaces in $\mathbf{C}^{2}$ by utilizing Legendre curves.

THEOREM 3.1. Let $f: I_{1} \rightarrow \mathbf{C}^{*}$ be a regular curve defined on an open interval $I_{1}$ and $z: I_{2} \rightarrow S^{3}(r) \subset \mathbf{C}^{2}$ be a regular spherical curve defined on an open interval $I_{2}$ containing 0 . Then, for any functions $p: I_{2} \rightarrow \mathbf{C}$, we have the following:

(i) If $z=z(t)$ is a Legendre curve, the map:

$$
L_{f p z}(s, t)=f(s) z(t)-\int_{0}^{t} p(t) z^{\prime}(t) d t
$$

defines a Lagrangian isometric immersion of $M^{2}=\left(U, g_{f p z}\right)$ into $\mathbf{C}^{2}$, where

$$
U=\left\{(s, t) \in I_{1} \times I_{2}: f(s) \neq p(t)\right\}
$$

and $g_{f p z}$ is the induced metric given by

$$
g_{f p z}=r^{2}\left|f^{\prime}(s)\right|^{2} d s^{2}+|f(s)-p(t)|^{2}\left|z^{\prime}(t)\right|^{2} d t^{2} .
$$

(ii) Conversely, if $f$ contains no circular arcs and if $L_{f p z}$ in (3.1) defines a Lagrangian immersion, then $z: I_{2} \rightarrow S^{3}(r) \subset \mathbf{C}^{2}$ is a Legendre curve.

Proof. Let $f: I_{1} \rightarrow \mathbf{C}^{*}$ be a regular curve, $z: I_{2} \rightarrow S^{3}(r) \subset \mathbf{C}^{2}$ be a regular spherical curve, and $L=L_{f p z}$ be the map defined by (3.1). Then we have

$$
\begin{aligned}
& L_{s}=\frac{\partial L}{\partial s}=f^{\prime}(s) z(t), \quad\left\langle z, z^{\prime}\right\rangle=0, \\
& L_{t}=\frac{\partial L}{\partial t}=(f(s)-p(t)) z^{\prime}(t) .
\end{aligned}
$$

By applying Lemma 2.2 and (3.3), we find

$$
\begin{aligned}
& \left\langle L_{s}, L_{s}\right\rangle=r^{2}\left|f^{\prime}(s)\right|^{2}, \\
& \left\langle L_{s}, L_{t}\right\rangle=\left\langle i f^{\prime}, f-p\right\rangle\left\langle z, i z^{\prime}\right\rangle, \\
& \left\langle L_{t}, L_{t}\right\rangle=|f-p|^{2}\left|z^{\prime}\right|^{2} .
\end{aligned}
$$

If $z(t)$ is a Legendre curve in $S^{3}(r) \subset \mathbf{C}^{2}$, then (3.3) and (3.4) imply that the induced metric on $U$ is given by (3.2). Since $z(t)$ is a Legendre curve, Lemma 2.2 and (3.3) imply $\left\langle L_{s}, i L_{t}\right\rangle=0$, which shows that $L: M^{2} \rightarrow \mathbf{C}^{2}$ is Lagrangian. This proves statement (i).

From (3.3) and Lemma 2.2, we have $\left\langle L_{s}, i L_{t}\right\rangle=\left\langle f^{\prime}, f-p\right\rangle\left\langle z, i z^{\prime}\right\rangle$. So, when $L_{f p z}$ is Lagrangian, we obtain $\left\langle f^{\prime}(s), f(s)-p(t)\right\rangle\left\langle z(t), i z^{\prime}(t)\right\rangle=0$ identically. 
If $\left\langle f^{\prime}(s), f(s)-p(t)\right\rangle=0$ holds for all $s$ in an open subinterval $I \subset I_{1}$, then we get $|f(s)-p(t)|_{s}^{2}=0$ which implies that, for each $t \in I_{2}$, the curve $f$ is contained in a circle centered at $p(t)$. This is impossible unless $p$ is a constant. Hence, when $f=f(s)$ contains no circular arcs, we get $\left\langle z, i z^{\prime}\right\rangle=0$, Thus, $z=$ $z(t)$ is a Legendre curve in $S^{3}(r)$.

Remark 3.1. For each $\mathbf{R} \ni b \neq 0$,

$$
z(t)=a\left(e^{i b t}, b e^{-i t / b}\right), \quad a=\frac{1}{\sqrt{1+b^{2}}},
$$

is a unit speed Legendre curve whose curvature $\lambda$ in $S^{3}(1)$ is the constant $\left(b^{2}-1\right) / b$. Unit speed Legendre curves in $S^{3}(1)$ with nonzero constant curvature are congruent to those curves. Obviously, such Legendre curves lie on a flat torus in $S^{3}(1)$. Moreover, when $b$ is a rational number the Legendre curve, $z(t)$ is a closed curve.

Remark 3.2. Using closed Legendre curves, we may construct many compact Lagrangian surfaces in $\mathbf{C}^{2}$ by applying Theorem 3.1. In fact, if $z=$ $z(t): S^{1}(a) \rightarrow S^{3}(1) \subset \mathbf{C}^{2}$ is a closed unit speed Legendre curve in $S^{3}(1) \subset \mathbf{C}^{2}$, $f=f(s): S^{1}(b) \rightarrow \mathbf{C}^{*}$ a constant speed curve in $\mathbf{C}^{*}$ and $p=p(t): S^{1}(a) \rightarrow \mathbf{C}$ a regular curve in $\mathbf{C}$ which does not intersects the curve $f$, then the immersion $L_{f p z}$ defined by (3.1) gives rise to a compact Lagrangian surface in $\mathbf{C}^{2}$ according to Theorem 3.1.

Remark 3.3. The Hopf fibration $\pi: S^{3}(1) \rightarrow C P^{1}(4)$ is given by

$$
\pi\left(z_{1}, z_{2}\right)=\frac{1}{2}\left(2 z_{1} \bar{z}_{2} ;\left|z_{1}\right|^{2}-\left|z_{2}\right|^{2}\right), \quad\left(z_{1}, z_{2}\right) \in S^{3}(1) \subset \mathbf{C}^{2} .
$$

For each Legendre curve $z=\left(z_{1}, z_{2}\right)$ in $S^{3}(1)$, the projection $\pi \circ z$ is a curve in $S^{2}\left(\frac{1}{2}\right)=C P^{1}(4)$. Conversely, each curve $\alpha$ in $S^{2}\left(\frac{1}{2}\right)$ gives rise to a horizontal lift $\tilde{\alpha}$ in $S^{3}(1)$ via $\pi$ which is unique up to a factor $e^{i r}, r \in \mathbf{R}$.

A horizontal lift of $\alpha$ in $S^{2}\left(\frac{1}{2}\right)$ is a Legendre curve in $S^{3}(1)$ with the same curvature function. For each complete curve $\alpha$ in $S^{2}\left(\frac{1}{2}\right)$, the horizontal lift $\tilde{\alpha}$ of $\alpha$ is a complete Legendre curve in $S^{3}(1)$. The complete Legendre curves obtained from closed curves in $S^{2}\left(\frac{1}{2}\right)$ via the Hopf fibration $\pi$ are closed Legendre curves if they were periodic. By using complete Legendre curves in $S^{3}(1)$, we can construct many complete Lagrangian surfaces in $\mathbf{C}^{2}$ via Theorem 3.1.

\section{Extrinsic properties}

For a unit speed plane curve $f: I_{1} \rightarrow \mathbf{C}$, we define the curvature function of $f$ by $\kappa(s)=\left\langle f^{\prime \prime}(s), i f^{\prime}(s)\right\rangle$. We may express $f$ in polar form as $f=\rho(s) e^{i \theta(s)}$.

THeOREM 4.1. Let $f: I_{1} \rightarrow \mathbf{C}^{*}$ be a unit speed curve, $z: I_{2} \rightarrow S^{3}(1) \subset \mathbf{C}^{2}$ a 
unit speed Legendre curve, $p=p(t)$ a complex-valued function defined on $I_{2}$, and $L=L_{f p z}:\left(U, g_{f p z}\right) \rightarrow \mathbf{C}^{2}$ be the Lagrangian isometric immersion defined by

$$
L_{f p z}(s, t)=f(s) z(t)-\int_{0}^{t} p(t) z^{\prime}(t) d t .
$$

Then we have:

(1) $L_{s}=\partial L / \partial s$ is an eigenvector of the shape operator $A_{J L_{s}}$ with eigenvalue $\kappa$, where $\kappa$ is the curvature function of the plane curve $f=f(s)$.

(2) $L_{t}=\partial L / \partial t$ is an eigenvector of the shape operator $A_{J L_{t}}$ if and only if $p$ is constant and $f(s)$ is a part of a line through the point $p$, i.e., $f(s)=$ $c s+p$ for some $c \in \mathbf{C}$ with $|c|=1$.

(3) $L$ is a totally geodesic immersion if and only if

(3.1) $p$ is constant, $f=f(s)$ is a part of a line through $p$, and

(3.2) $z: I_{2} \rightarrow S^{3}(1) \subset \mathbf{C}^{2}$ is a great circle in $S^{3}(1)$.

Proof. Under the hypothesis, we know from (3.2) of Theorem 3.1 that the induced metric on $U$ via $L_{f p z}$ is the twisted product metric:

$$
g_{f p z}=d s^{2}+|f(s)-p(t)|^{2} d t^{2} .
$$

From (4.1) we have

$$
\begin{aligned}
L_{s} & =\frac{\partial L}{\partial s}=f^{\prime}(s) z(t), \\
L_{t} & =\frac{\partial L}{\partial t}=(f(s)-p(t)) z^{\prime}(t), \\
L_{s s} & =f^{\prime \prime}(s) z(t), \quad L_{s t}=f^{\prime}(s) z^{\prime}(t), \\
L_{t t} & =(f(s)-p(t)) z^{\prime \prime}(t)-p^{\prime}(t) z^{\prime}(t) .
\end{aligned}
$$

By applying (2.3) and (4.3), we find

$$
\begin{aligned}
& \left\langle L_{s s}, i L_{s}\right\rangle=\left\langle f^{\prime \prime}, i f^{\prime}\right\rangle=\kappa, \\
& \left\langle L_{s s}, i L_{t}\right\rangle=\left\langle L_{s t}, i L_{s}\right\rangle=0, \\
& \left\langle L_{t t}, i L_{s}\right\rangle=\left\langle L_{s t}, i L_{t}\right\rangle=\left\langle f^{\prime}, i f-i p\right\rangle, \\
& \left\langle L_{t t}, i L_{t}\right\rangle=|f-p|^{2}\left\langle z^{\prime \prime}, i z^{\prime}\right\rangle-\left\langle p^{\prime}, i f-i p\right\rangle .
\end{aligned}
$$

Thus, we obtain statement (1) by applying (4.2) and (4.4).

From the third equation in (4.4), we know that $L_{t}=\partial L / \partial t$ is an eigenvector of $A_{J L_{t}}$ if and only if $\left\langle f^{\prime}(s)\right.$, if $\left.(s)-i p(t)\right\rangle=0$ holds identically. Since the later condition holds if and only if the position vector of $\gamma_{p(t)}(s)=f(s)-p(t)$ is always tangent to the curve $\gamma_{p(t)}$ for any fixed $t$. Therefore, for each $t, \gamma_{p(t)}$ is a part of a line through the origin of $\mathbf{C}$. Thus, there exists a unit vector field $c(t)$ in $\mathbf{C}$ such that $f(s)-p(t)=c(t) s$. This implies that $c$ and $p$ are constant. Thus, we get $f(s)=c s+p$, Therefore, $f(s)$ is a part of a line through $p$. This gives statement (2). 
Let us put $e_{1}=L_{s}$ and $e_{2}=L_{t} /|f-p|$. Then $e_{1}, e_{2}$ are orthonormal vector fields according to (4.2). Hence, by using (4.2), (4.4) and Lemma 2.1, we have

$$
\begin{aligned}
A_{J e_{1}} & =\left(\begin{array}{cc}
\kappa(s) & 0 \\
0 & \frac{\left\langle f^{\prime}, i f-i p\right\rangle}{|f-p|^{2}}
\end{array}\right), \\
A_{J e_{2}} & =\left(\begin{array}{cc}
0 & \frac{\left\langle f^{\prime}, i f-i p\right\rangle}{|f-p|^{2}} \\
\frac{\left\langle f^{\prime}, i f-i p\right\rangle}{|f-p|^{2}} & \frac{\lambda(t)}{|f-p|}-\frac{\left\langle p^{\prime}, i f-i p\right\rangle}{|f-p|^{3}}
\end{array}\right),
\end{aligned}
$$

where $\lambda$ is the curvature function of $z$ in $S^{3}(1)$.

Suppose that $L: M^{2} \rightarrow \mathbf{C}^{2}$ is a totally geodesic immersion. Then, statement (2) implies that $p$ is constant and $f=c s+p$ for some unit complex number $c$. Moreover, from (4.5), we also have

$$
0=\lambda(t)|f(s)-p|^{2}-\left\langle p^{\prime}, \text { if }(s)-i p\right\rangle=\lambda(t)|f(s)-p|^{2} .
$$

Using these we find $\lambda=0$. This shows that the Legendre curve $z$ has zero curvature in $S^{3}(1)$. Thus, $z$ is an open portion of a Legendre great circle in $S^{3}(1)$.

The converse follows easily from (4.4) and Lemma 2.1. Thus, we obtain statement (3).

\section{Minimality}

The Lagrangian surface $M_{0}$ in $\mathbf{C}^{2}$ defined by

$$
M_{0}:=\left\{\left(z_{1}, z_{2}\right) \in \mathbf{C}^{2}:\left|z_{1}\right| z_{2}=\left|z_{2}\right| z_{1} \text { and } 2\left|z_{1}\right|\left|z_{2}\right|=1\right\}
$$

is an area-minimizing minimal Lagrangian surface invariant under the diagonal action of $S O(2)$ which is known as the Lagrangian catenoid (see [21]). It was proved in [4] that, up to dilations, the Lagrangian catenoid is the only minimal non-flat Lagrangian surface of revolution in $\mathbf{C}^{2}$. Moreover, it was shown in [5] that, up to dilations, the Lagrangian catenoid is the only minimal non-flat Lagrangian surface which is foliated by circles of $\mathbf{C}^{2}$.

It is well-known that an orientable minimal surface in $\mathbf{C}^{2}=\left(\mathbf{E}^{4}, J\right)$ is a Lagrangian surface if and only if it is a holomorphic curve with respect to some other orthogonal almost complex structure on $\mathbf{E}^{4}$ ([19], also see [2] for a simple alternate proof of this fact). The holomorphic curve corresponding to the Lagrangian catenoid is $(w, 1 / w), w \in \mathbf{C}^{*}$.

THeOREM 5.1. Let $f: I_{1} \rightarrow \mathbf{C}^{*}$ be a unit speed curve, $z: I_{2} \rightarrow S^{3}(1) \subset \mathbf{C}^{2}$ a unit speed Legendre curve, and $p=p(t)$ a complex-valued function defined on $I_{2}$. Then the Lagrangian immersion $L_{f p z}:\left(U, g_{f p z}\right) \rightarrow \mathbf{C}^{2}$ defined by 


$$
L_{f p z}(s, t)=f(s) z(t)-\int_{0}^{t} p(t) z^{\prime}(t) d t
$$

is minimal if and only if either $L_{f p z}$ is a totally geodesic Lagrangian surface or, up to dilations and rigid motions, $L_{f p z}$ is an open portion of the Lagrangian catenoid.

Proof. Let $f: I_{1} \rightarrow \mathbf{C}^{*}$ be a unit speed curve, $z: I_{2} \rightarrow S^{3}(1) \subset \mathbf{C}^{2}$ a unit speed Legendre curve, and $p=p(t)$ a complex-valued function defined on $I_{2}$. Then (4.5) and Theorem 4.1 imply that $L_{f p z}$ is a minimal Lagrangian immersion if and only if we have:

(i) The curvature function $\kappa(s)$ of $f$ satisfies

$$
\kappa(s)|f(s)-p(t)|^{2}+\left\langle f^{\prime}(s), \text { if }(s)-i p(t)\right\rangle=0 .
$$

(ii) The curvature function $\lambda(t)$ of the Legendre curve $z(t)$ in $S^{3}(1)$ satisfies

$$
\lambda(t)|f(s)-p(t)|^{2}-\left\langle p^{\prime}(t), \text { if }(s)-i p(t)\right\rangle=0 .
$$

Now, let us assume that $L_{f p z}$ is a minimal Lagrangian immersion.

CASE (a). $f^{\prime \prime}(s)=0$. In this case we have $\kappa=0$ and $f(s)=c_{1} s+c_{2}$ for some complex numbers $c_{1}, c_{2}$ with $\left|c_{1}\right|=1$. Also from (5.2) we get $\left\langle f^{\prime}\right.$, if $\left.-i p\right\rangle$ $=0$. Thus, by applying (4.5) and (5.3), we know that $L_{f p z}$ is a totally geodesic immersion.

CASE (b). $\quad f^{\prime \prime}(s) \neq 0 . \quad$ In this case we have $\kappa \neq 0$ since $f^{\prime \prime}=i \kappa f^{\prime} . \quad$ By differentiating (5.2) with respect to $s$, we find

$$
\kappa^{\prime}(s)|f(s)-p(t)|^{2}+3 \kappa\left\langle f^{\prime}(s), f(s)-p(t)\right\rangle=0,
$$

which can be expressed as

$$
(\ln |\kappa(s)|)^{\prime}+\frac{3}{2} \frac{\partial}{\partial s}\left(\ln |f-p|^{2}\right)=0 .
$$

By solving (5.4) we get

$$
|f-p|^{2}=\frac{\psi^{2}(t)}{\kappa^{2 / 3}(s)}
$$

for some real-valued function $\psi(t)$. Substituting (5.5) into (5.2) gives

$$
\left\langle f(s)-p(t), i f^{\prime}(s)\right\rangle=\psi^{2}(t) \kappa^{1 / 3}(s) .
$$

Differentiating (5.6) with respect to $s$ gives

$$
\left\langle f(s)-p(t), f^{\prime}(s)\right\rangle=-\frac{\kappa^{\prime} \psi^{2}(t)}{3 \kappa^{5 / 3}} .
$$


Since $f$ is a unit speed curve with $\kappa \neq 0, f^{\prime}(s), f^{\prime \prime}(s) / \kappa$ form an orthonormal frame over R. Thus, we obtain from (5.6) and (5.7) that

$$
f(s)=p(t)-\kappa^{1 / 3}\left(\frac{\kappa^{\prime}}{3 \kappa^{2}}-i\right) \psi^{2}(t) f^{\prime}(s) .
$$

By differentiating (5.8) with respect to $t$, we find

$$
p^{\prime}(t)=2 \kappa^{1 / 3}\left(\frac{\kappa^{\prime}}{3 \kappa^{2}}-i\right) \psi(t) \psi^{\prime}(t) f^{\prime}(s)
$$

If $\psi^{\prime}(t) \neq 0$, then $(5.9)$ gives

$$
\frac{p^{\prime}(t)}{\psi(t) \psi^{\prime}(t)}=2 \kappa^{1 / 3}\left(\frac{\kappa^{\prime}}{3 \kappa^{2}}-i\right) f^{\prime}(s)
$$

which implies that

$$
p^{\prime}(t)=2 \delta \psi(t) \psi^{\prime}(t), \quad\left(\frac{\kappa^{\prime}}{3 \kappa^{5 / 3}}-i \kappa^{1 / 3}\right) f^{\prime}(s)=\delta
$$

for some complex number $\delta$. Thus, we have

$$
p(t)=\psi^{2}(t) \delta+\eta
$$

for some complex numbers $\delta, \eta$. Substituting (5.12) into (5.8) gives

$$
f(s)=\left\{\delta-\left(\frac{\kappa^{\prime}}{3 \kappa^{5 / 3}}-i \kappa^{1 / 3}\right) f^{\prime}(s)\right\} \psi^{2}(t)+\eta,
$$

which implies that $\psi$ is constant. Thus, $p$ is also constant by (5.12). Hence, $\lambda(t)=0$ by (5.3). Therefore, $z=z(t)$ is a Legendre great circle in $S^{3}(1)$. Moreover, from (5.5), we find

$$
|f(s)-p|^{2}=\left(\frac{a^{2}}{\kappa(s)}\right)^{2 / 3}
$$

where $p \in \mathbf{C}$ and $0 \neq a \in \mathbf{R}$. Substituting (5.14) into (5.2) gives

$$
\left\langle i f^{\prime}(s), f(s)-p\right\rangle=a^{4 / 3} \kappa^{1 / 3} \text {. }
$$

Now, let us reparametrize $f$ as $f(s(x))=p+x+i y(x)$. Then we have

$$
\kappa=\frac{y^{\prime \prime}(x)}{\left(1+y^{\prime}(x)^{2}\right)^{3 / 2}}, \quad\left\langle i f^{\prime}, f-p\right\rangle=\frac{y-x y^{\prime}(x)}{\left(1+y^{\prime}(x)^{2}\right)^{1 / 2}} .
$$

By combining (5.15) and (5.16), we get

$$
a^{4} y^{\prime \prime}(x)=\left(y-x y^{\prime}(x)\right)^{3} .
$$

Let $u=y-x y^{\prime}$. We get $d u / d x=-x y^{\prime \prime}$. Thus, (5.17) becomes $a^{4} u^{\prime}(x)=-x u^{3}$. Solving this equation gives $y-x y^{\prime}= \pm a^{2} / \sqrt{x^{2}-a^{4} c}$ for some constant $c$. After solving this first order differential equation, we have 


$$
y=\mp\left(\frac{\sqrt{x^{2}-a^{4} c}}{a^{2} c}+b x\right)
$$

for some real number $b$. Thus, we find

$$
f=p+(1 \mp i b) x \mp i \frac{\sqrt{x^{2}-a^{4} c}}{a^{2} c} .
$$

From (5.16) and (5.19), we discover that

$$
\kappa=\frac{ \pm a^{8} c^{3}}{\left(\left(x+a^{2} b c \sqrt{x^{2}-a^{4} c}\right)^{2}+a^{4} c^{2}\left(x^{2}-a^{4} c\right)\right)^{3 / 2}} .
$$

Hence, we obtain from (5.14), (5.19) and (5.20) that

$$
\left(x+a^{2} b c \sqrt{x^{2}-a^{4} c}\right)^{2}+a^{4} c^{2}\left(x^{2}-a^{4} c\right)=a^{4} c^{2}\left(x^{2}+\left(\frac{\sqrt{x^{2}-a^{4} c}}{a^{2} c}+b x\right)^{2}\right),
$$

which implies that $b^{2}=\left(1-a^{4} c^{2}\right) /\left(a^{4} c^{2}\right)$. Thus, we have $a^{4} c^{2} \leq 1$. Consequently, we see from (5.18) that $f(s)-p=x(s)+i y(s)$ lies in the hyperbola:

$$
x^{2} \pm \frac{2 \sqrt{1-a^{4} c^{2}}}{a^{2} c} x y-y^{2}=\frac{1}{c} .
$$

By applying the following rotation:

$$
\begin{aligned}
& x=\cos \left(\frac{1}{2} \tan ^{-1}\left(\frac{\sqrt{1-a^{4} c^{2}}}{a^{2} c}\right)\right) \tilde{x} \mp \sin \left(\frac{1}{2} \tan ^{-1}\left(\frac{\sqrt{1-a^{4} c^{2}}}{a^{2} c}\right)\right) \tilde{y}, \\
& y= \pm \sin \left(\frac{1}{2} \tan ^{-1}\left(\frac{\sqrt{1-a^{4} c^{2}}}{a^{2} c}\right)\right) \tilde{x}+\cos \left(\frac{1}{2} \tan ^{-1}\left(\frac{\sqrt{1-a^{4} c^{2}}}{a^{2} c}\right)\right) \tilde{y},
\end{aligned}
$$

on $\mathbf{C}^{2}$, equation (5.21) becomes $\tilde{x}^{2}-\tilde{y}^{2}=a^{2}$. Since $z(t)$ is a unit speed Legendre great circle in $S^{3}(1) \subset \mathbf{C}^{2}$ which can be represented by $(1 / \sqrt{2})\left(e^{i t}, e^{-i t}\right)$, the Lagrangian immersion $L_{f p z}$ is thus congruent to $\left(a e^{u} e^{i s}, a e^{-u} e^{-i s}\right)$ in $\mathbf{E}^{4}$. Thus, up to dilations and rigid motions, $L_{f p z}$ is an open portion of the Lagrangian catenoid $M_{0}$.

The converse can be verified by direct computation.

\section{Hamiltonian minimality}

Oh [26] introduced the notion of Hamiltonian minimal surfaces as follows. A Lagrangian immersion $L: M \rightarrow \mathbf{C}^{2}$ is said to be Hamiltonian minimal if it is a critical point of the area functional restricted to (compactly supported) Hamiltonian variations of $L$, i.e., variations with normal variational vector field $\xi$ such that the dual 1 -form $\alpha_{\xi}$ of $J \xi$ on $M$ is exact. 
Lemma 6.1. If $f=f(s): I_{1} \rightarrow \mathbf{C}^{*}$ is a unit speed curve, $z=z(t): I_{2} \rightarrow$ $S^{3}(1) \subset \mathbf{C}^{2}$ a unit speed Legendre curve, and $p=p(t)$ a complex-valued function defined on $I_{2}$, then the Lagrangian immersion $L_{f p z}:\left(U, g_{f p z}\right) \rightarrow \mathbf{C}^{2}$ is Hamiltonian minimal if and only if we have

$$
\begin{aligned}
\kappa^{\prime}(s) \mid f & -\left.p\right|^{6}+\left\{\lambda^{\prime}(t)+2 \kappa(s)\left\langle f^{\prime}, f-p\right\rangle\right\}|f-p|^{4} \\
& +\left\langle\lambda p^{\prime}+i p^{\prime \prime}+\left\langle i f^{\prime}, f-p\right\rangle f^{\prime}, f-p\right\rangle|f-p|^{2} \\
= & 3\left\langle i p^{\prime}, p-f\right\rangle\left\langle p^{\prime}, f-p\right\rangle,
\end{aligned}
$$

where $\kappa$ is the curvature of $f$ in $\mathbf{C}$ and $\lambda$ is the curvature of $z$ in $S^{3}(1)$.

Proof. It follows from (4.2) and (4.5) that $\alpha_{H}$ satisfies

$$
-2 \alpha_{H}=\left(\kappa(s)+\frac{\left\langle f^{\prime}, i f-i p\right\rangle}{|f-p|^{2}}\right) d s+\left(\lambda(t)-\frac{\left\langle p^{\prime}, i f-i p\right\rangle}{|f-p|^{2}}\right) d t .
$$

In views of (4.2), we know that $e_{1}=\partial / \partial s$ and $e_{2}=|f-p|^{-1} \partial / \partial t$ form an orthonormal frame. So, the dual frame of $\left\{e_{1}, e_{2}\right\}$ is given by

$$
\omega^{1}=d s, \quad \omega^{2}=|f-p| d t .
$$

From these we find

$$
d \omega^{1}=0, \quad d \omega^{2}=\frac{\left\langle f^{\prime}, f-p\right\rangle}{|f-p|^{2}} \omega^{1} \wedge \omega^{2} .
$$

If $*$ denotes the star operator on $M$, we obtain from (6.2) that

$$
2\left(* \alpha_{H}\right)=\left(\frac{\lambda(t)}{|f-p|}-\frac{\left\langle p^{\prime}, i f-i p\right\rangle}{|f-p|^{3}}\right) \omega^{1}-\left(\kappa(s)+\frac{\left\langle f^{\prime}, i f-i p\right\rangle}{|f-p|^{2}}\right) \omega^{2} .
$$

Therefore, by applying $f^{\prime \prime}(s)=i \kappa(s) f^{\prime}(s)$ and (6.3), we know that Maslov 1-form $\alpha_{H}$ is co-closed if and only if (6.1) holds identically.

On the other hand, it is also known from [26] that a Lagrangian immersion $L: M \rightarrow \mathbf{C}^{2}$ is Hamiltonian minimal if and only if its Maslov form is co-closed. Therefore, we obtain the lemma by combining these two results.

Lemma 6.2. If $f: I_{1} \rightarrow \mathbf{C}^{*}$ is a unit speed curve and $z: I_{2} \rightarrow S^{3}(1) \subset \mathbf{C}^{2}$ a unit speed Legendre curve, then the immersion

$$
L_{f z}: I_{1} \times I_{2} \rightarrow \mathbf{C}^{2} ; \quad(s, t) \mapsto f(s) z(t)
$$

is a Hamiltonian minimal Lagrangian immersion (with respect to the induced metric) if and only if the following two conditions hold: 

$\alpha \in \mathbf{R}$.

(i) The curvature function $\lambda(t)$ of $z(t)$ in $S^{3}(1)$ satisfies $\lambda^{\prime}(t)=\alpha$ for some

(ii) The curvature function $\kappa(s)$ of $f(s)$ satisfies either

$$
\kappa^{\prime}(s) F^{2}(s)+\left(\alpha+\kappa(s) F^{\prime}(s)\right) F(s)=\frac{F^{\prime}(s)}{4} \sqrt{4 F(s)-F^{\prime 2}(s)},
$$

or

$$
\kappa^{\prime}(s) F^{2}(s)+\left(\alpha+\kappa(s) F^{\prime}(s)\right) F(s)=-\frac{F^{\prime}(s)}{4} \sqrt{4 F(s)-F^{\prime 2}(s)},
$$

where $F(s)=|f(s)|^{2}$.

Proof. Let $f: I_{1} \rightarrow \mathbf{C}^{*}$ be a unit speed curve and let $z: I_{2} \rightarrow S^{3}(1) \subset \mathbf{C}^{2}$ be a unit speed Legendre curve. Assume that the Lagrangian immersion $L_{f z}(s, t)=$ $f(s) z(t)$ is Hamiltonian minimal. Then Lemma 6.1 implies that the curvature $\kappa$ of $f(s)$ and the curvature of $z(t)$ in $S^{3}(1)$ satisfy

$$
\kappa^{\prime}|f|^{4}+\left\{\lambda^{\prime}(t)+2 \kappa\left\langle f^{\prime}, f\right\rangle\right\}|f|^{2}=\left\langle f^{\prime}, f\right\rangle\left\langle f^{\prime}, \text { if }\right\rangle .
$$

Since both $\kappa$ and $f$ are independent of $t,(6.8)$ implies that $\lambda^{\prime}(t)$ is a real number, say $\alpha$. So, we obtain condition (i).

Because $f(s)$ is assumed to be of unit speed, we have

$$
f=\left\langle f, f^{\prime}\right\rangle f^{\prime}+\left\langle f, i f^{\prime}\right\rangle i f^{\prime}
$$

which implies that

$$
\left\langle f, i f^{\prime}\right\rangle=\mp \frac{1}{2} \sqrt{4 F-F^{\prime 2}(s)} .
$$

If $\left\langle f, i f^{\prime}\right\rangle=-\frac{1}{2} \sqrt{4 F-F^{\prime 2}(s)}$ holds, then we obtain (6.6). Similarly, if we have $\left\langle f, i f^{\prime}\right\rangle=\frac{1}{2} \sqrt{4 F-F^{\prime 2}(s)}$, then we obtain (6.7).

Conversely, by applying Lemma 6.1 , it is easy to verify that conditions (i) and (ii) imply the Hamiltonian minimality of the Lagrangian immersion $L_{f z}$.

The following theorem determines Hamiltonian minimal Lagrangian immersions defined by $L_{f z}$ such that $f$ does not contained in a line through the origin.

THEOREM 6.1. Let $f: I_{1} \rightarrow \mathbf{C}^{*}$ be a unit speed curve defined on an open interval $I_{1} \ni 0$ and $z: I_{2} \rightarrow S^{3}(1) \subset \mathbf{C}^{2}$ be a unit speed Legendre curve. If $f(s)$ is not parallel to $f^{\prime}(s)$ for $s \in I_{1}$, then $L_{f z}$ defined by (6.5) is Hamiltonian minimal if and only if we have:

(a) The curvature function $\lambda$ of $z$ in $S^{3}(1)$ is given by $\lambda(t)=\alpha t+\beta$ for some $\alpha, \beta \in \mathbf{R} ;$ and 
(b) Up to rotations about the origin, the curve $f(s)$ is given by

$$
f(s)=\sqrt{F(s)} \exp \left( \pm \frac{i}{2} \int_{0}^{s} \frac{\sqrt{4 F(s)-F^{\prime 2}(s)}}{F(s)} d s\right),
$$

where $F(s)$ with $4 F(s)>F^{\prime}(s)^{2}$ is a positive solution of the differential equation:

$$
4 F^{2}\left(4 F-F^{2}\right) F^{\prime \prime \prime}+F^{\prime}\left(F^{2}-2 F F^{\prime \prime}\right)^{2}= \pm 4 \alpha F\left(4 F-F^{\prime 2}\right)^{3 / 2} .
$$

Proof. Let $f: I_{1} \rightarrow \mathbf{C}^{*}$ be a unit speed curve defined on an open interval $I_{1} \ni 0$ and let $z: I_{2} \rightarrow S^{3}(1) \subset \mathbf{C}^{2}$ be a unit speed Legendre curve. Assume that $f(s)$ is not parallel to $f^{\prime}(s)$ for any $s \in I_{1}$. Then $\left\langle f, i f^{\prime}\right\rangle$ is nowhere zero.

If $L_{f z}(s, t)=f(s) z(t)$ is Hamiltonian minimal, then Lemma 6.2 implies that $z$ has constant curvature in $S^{3}(1)$ and $f$ satisfies condition (ii) of Lemma 6.2.

By using the assumption that $f(s)$ is of unit speed, we find

$$
f^{\prime \prime}(s)=i \kappa(s) f^{\prime}(s), \quad f=\left\langle f, f^{\prime}\right\rangle f^{\prime}+\left\langle f, i f^{\prime}\right\rangle i f^{\prime} .
$$

So, by applying the first equation in (6.12), we find

$$
F^{\prime \prime}=2+2 \kappa\left\langle f, i f^{\prime}\right\rangle,
$$

where $F=|f|^{2}$. Hence, from (6.9) and the second equation in (6.12), we obtain

$$
\kappa(s)= \pm \frac{2-F^{\prime \prime}(s)}{\sqrt{4 F(s)-F^{\prime 2}(s)}} .
$$

By substituting (6.13) into (6.6) and (6.7), we obtain (6.11). Due to $F^{\prime}=2\left\langle f, f^{\prime}\right\rangle$ and (6.9), the second equation in (6.12) can be expressed as

$$
f^{\prime}(s)=\frac{F^{\prime}(s) \pm i \sqrt{4 F(s)-F^{\prime 2}(s)}}{2 F(s)} f(s) .
$$

After solving this differential equation we obtain

$$
f(s)=\gamma \sqrt{F(s)} \exp \left( \pm \frac{i}{2} \int_{0}^{s} \frac{\sqrt{4 F(s)-F^{\prime 2}(s)}}{F(s)} d s\right)
$$

for some complex number $\gamma$.

Now, by a direct computation we obtain $\left|f^{\prime}(s)\right|^{2}=|\gamma|^{2}$. Because $f(s)$ is of unit speed, this implies that $\gamma$ is unitary. Hence, after applying a suitable rotation about the origin in $\mathbf{C}$, we have $\gamma=1$. Consequently, up to rotations of $\mathbf{C}$ about the origin, $f(s)$ is given by $(6.10)$.

Conversely, assume that $F(s)$ is a positive solution of the differential equation (6.11) and it satisfies $4 F>F^{\prime 2}$. Let us define the curve $f(s)$ in $\mathbf{C}^{*}$ by (6.10). Then we have 


$$
\begin{aligned}
F(s) & =|f(s)|^{2}, \\
f^{\prime}(s) & =\frac{F^{\prime}(s) \pm i \sqrt{4 F(s)-F^{\prime 2}(s)}}{2 \sqrt{F(s)}} \exp \left(\frac{i}{2} \int_{0}^{s} \frac{\sqrt{4 F-F^{\prime 2}}}{F} d s\right), \\
f^{\prime \prime}(s) & =\frac{\left(2-F^{\prime \prime}\right)\left( \pm i F^{\prime}-\sqrt{4 F-F^{\prime 2}}\right)}{2 \sqrt{4 F^{2}-F F^{\prime 2}}} \exp \left(\frac{i}{2} \int_{0}^{s} \frac{\sqrt{4 F-F^{\prime 2}}}{F} d s\right) .
\end{aligned}
$$

It follows from (6.17) that $\left|f^{\prime}(s)\right|=1$. Hence $f(s)$ is a unit speed curve. From (6.17), (6.18) and the identity $f^{\prime \prime}(s)=i \kappa(s) f^{\prime}(s)$ we know that the curvature of $f(s)$ is given by (6.12). Thus, (6.11) can be expressed as one of (6.6) and (6.7). From these, we conclude that the Lagrangian immersion $L_{f z}$ is Hamiltonian minimal according to Lemma 6.2.

Remark 6.1. The third order differential equation (6.11) admits infinitely many positive solutions $F(s)$ with $4 F(s)>F^{\prime 2}(s)$. This can be seen as follows:

Let us put $y_{1}=F(s), y_{2}=F^{\prime}(s)$ and $y_{3}=F^{\prime \prime}(s)$. Then (6.11) is equivalent to the following first order differential system:

$$
\begin{aligned}
& y_{1}^{\prime}(s)=y_{2}, \quad y_{2}^{\prime}(s)=y_{3}, \\
& y_{3}^{\prime}(s)=\frac{y_{2}\left(y_{2}^{2}-2 y_{1} y_{3}\right)^{2}}{4 y_{1}^{2}\left(y_{2}^{2}-4 y_{1}\right)} \pm \frac{\alpha \sqrt{4 y_{1}-y_{2}^{2}}}{y_{1}} .
\end{aligned}
$$

It follows from Picard's theorem (see, for instance [25, page 819]) that, for any given initial conditions:

$$
y_{1}\left(s_{0}\right)=y_{1}^{0}, \quad y_{2}\left(s_{0}\right)=y_{2}^{0}, \quad y_{3}\left(s_{0}\right)=y_{3}^{0}
$$

at an initial point $s_{0}$ with $y_{1}^{0}>0$ and $4 y_{1}^{0}>y_{2}^{0}$, the initial value problem has a unique solution in some interval containing $s_{0}$. Therefore, the third order differential equation (6.11) admits infinitely many positive solutions $F(s)$ with $4 F(s)>F^{\prime 2}(s)$.

Remark 6.2. If $F^{\prime \prime}(s) \neq 2$ (i.e., the curvature function of $f(s)$ is nonzero), then the third order differential equation (6.11) with $\alpha=0$ can be written as

$$
2\left(\frac{F^{2}\left(2-F^{\prime \prime}\right)^{2}}{4 F-F^{\prime 2}}\right)^{\prime}=2 F^{\prime}-F^{\prime} F^{\prime \prime},
$$

which is equivalent to

$$
4 F^{2}\left(2-F^{\prime \prime}\right)^{2}=(4 F-w)(4 F-w+c),
$$

where $c$ is a real number. Equation (6.21) can be written as

$$
4 F^{2}\left(2-F^{\prime} \frac{d F^{\prime}}{d F}\right)^{2}=\left(4 F-F^{2}\right)\left(4 F-F^{\prime 2}+c\right) .
$$


Solving (6.22) for $F^{\prime}$ yields

$$
F^{\prime 2}=4 F-\frac{(F-c r)^{2}}{4 r F} \text { or } \quad F^{\prime 2}=4 F-\frac{(c F-r)^{2}}{4 r F}
$$

for some positive number $r$. Therefore, if $\varphi(F)$ is an anti-derivative of

$$
\frac{2 \sqrt{r F}}{\sqrt{16 r F^{2}-(F-c r)^{2}}} \text { or } \frac{2 \sqrt{r F}}{\sqrt{16 r F^{2}-(c F-r)^{2}}} \text {, }
$$

then the inverse function $F(s)=\varphi^{-1}(s)$ of $\varphi(F)$ is a solution of (6.23). Hence, it is also a solution of the third order nonlinear equation (6.11) with $\alpha=0$.

Remark 6.3. In principle, one can construct infinitely many examples of Hamiltonian minimal Lagrangian surfaces by using the solutions of differential equation (6.11) via Theorem 6.1. Here we provide two families of such Hamiltonian minimal Lagrangian surfaces.

Example 6.1. For any $c \in \mathbf{R}$, a unit speed Legendre curve in $S^{3}(1)$ with curvature $c$ is congruent to

$$
z(t)=\left(1 / \sqrt{1+b^{2}}\right)\left(e^{i b t}, b e^{-i t / b}\right)
$$

with $b=\frac{1}{2}\left(c+\sqrt{4+c^{2}}\right)$.

On the other hand, the simplest solution of the differential equation (6.11) with $\alpha=0$ is given by $F(s)=r^{2}$, where $r$ is an arbitrary positive number. The unit speed curve $f(s)$ in $(6.10)$ corresponding to $F(s)=r^{2}$ is the circle given by $f(s)=r e^{i s / r}$. Therefore, the corresponding Hamiltonian minimal immersion $L_{f z}$ is congruent to

$$
L_{f z_{b}}(s, t)=\frac{r e^{i s / r}}{\sqrt{1+b^{2}}}\left(e^{i b t}, b e^{-i t / b}\right) .
$$

By applying formula (7.1), we know that, for any real numbers $r, b>0$, this Hamiltonian minimal Lagrangian surface is flat.

Example 6.2. If we put $r=1 / 4 \sqrt{1-a^{2}}$ for some $a \in(0,1)$, then $\varphi(F)=$ $\sqrt{F} / a$ is an anti-derivative of the first function in (6.24) with $c=0$. Thus, the inverse function $F(s)=a^{2} s^{2}$ is a solution of (6.11) with $\alpha=0$.

It is easy to verify that the unit speed curve $f(s)$ corresponding to $F(s)=$ $a^{2} s^{2}$ is $f(s)=a s^{1+i \sqrt{1-a^{2}} / a}$ and the curvature function of $f(s)$ is $\sqrt{1-a^{2}} /(a s)$.

The corresponding Hamiltonian minimal immersion $L_{f z}$ is thus congruent to

$$
L_{f z}(s, t)=\frac{a s^{1+i a^{-1} \sqrt{1-a^{2}}}}{\sqrt{1+b^{2}}}\left(e^{i b t}, b e^{-i t / b}\right) .
$$

For any real numbers $a, c>0$, this Hamiltonian minimal Lagrangian surface is flat. 


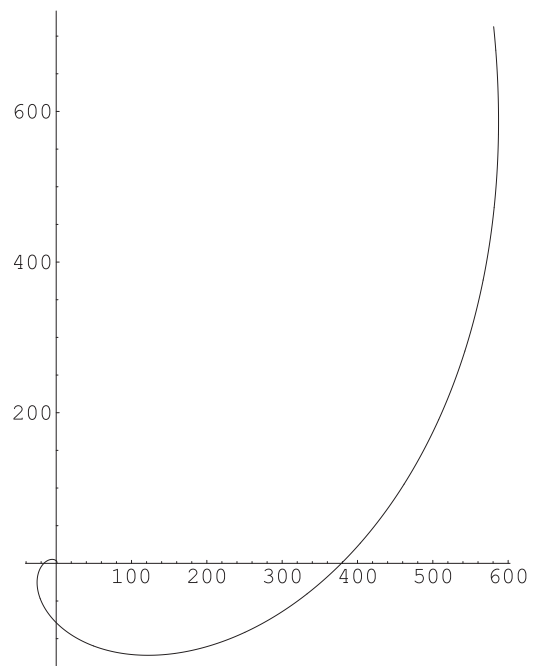

Figure 1. Curve $f(s)=s^{1+i} / \sqrt{2}$.

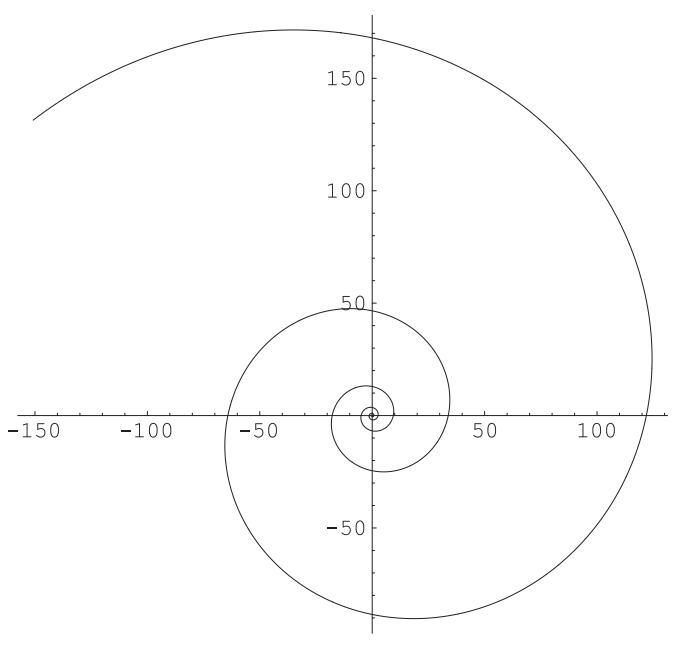

FIGURE 2. Curve $f(s)=s^{1+2 \sqrt{6} i} / 5$.

Remark 6.4. In general, the Hamiltonian stationary Lagrangian surfaces given in section 4 of [3] seems to be different from the examples given above, since our examples of Hamiltonian Lagrangian surfaces do not admit a circle symmetry in general. However, the Hamiltonian stationary Lagrangian surfaces $f_{0, a}(0<a<1)$ given in Remark 4 of [3] are congruent to the Lagrangian surfaces $L_{f z_{b}}$ defined by (6.25) with $b=1$ (i.e., to those with $z$ being a Legendre great circle in $\left.S^{3}(1)\right)$.

Remark 6.5. Hamiltonian stationary tori in $\mathbf{C}^{2}$ are also studied and constructed by Hélein and Ramon in [22] which include the examples previously constructed by Castro and Urbano in [6].

Corollary 6.1. If $f: I_{1} \rightarrow \mathbf{C}^{*}$ is a unit speed curve with constant curvature $\kappa$ and $z: I_{2} \rightarrow S^{3}(1) \subset \mathbf{C}^{2}$ a unit speed Legendre curve, then the Lagrangian immersion $L_{f z}$ defined by $L_{f z}=f(s) z(t)$ is Hamiltonian minimal if and only if we have

(i) $z: I_{2} \rightarrow S^{3}(1)$ has constant curvature in $S^{3}(1)$ and

(ii) $f$ is either an open portion of a line through the origin or an open portion of a circle centered at the origin.

Proof. Let $f: I_{1} \rightarrow \mathbf{C}^{*}$ be a unit speed curve with constant curvature $\kappa$ and $z: I_{2} \rightarrow S^{3}(1) \subset \mathbf{C}^{2}$ a unit speed Legendre curve in $S^{3}(1)$. Assume that the Lagrangian surface defined by $L_{f z}(s, t)=f(s) z(t)$ is Hamiltonian minimal.

If $\kappa=0$, we have $f(s)=c_{1} s+c_{2}$ for some unitary complex number $c_{1}$ and complex number $c_{2}$. Thus we obtain 


$$
F(s)=s^{2}+2\left\langle c_{1}, c_{2}\right\rangle s+\left|c_{2}\right|^{2} .
$$

Substituting this into (6.6) or (6.7) of Lemma 6.2 gives

$$
\alpha\left(s^{2}+2\left\langle c_{1}, c_{2}\right\rangle s+\left|c_{2}\right|^{2}\right)= \pm \sqrt{\left|c_{2}\right|^{2}-\left\langle c_{1}, c_{2}\right\rangle^{2}}\left(s+\left\langle c_{1}, c_{2}\right\rangle\right),
$$

which implies $\alpha=0$ and $\left|c_{2}\right|= \pm\left\langle c_{1}, c_{2}\right\rangle$. From $\alpha=0$ we see that $z$ has constant curvature in $S^{3}(1)$. Since $c_{1}$ is unitary, $\left|c_{2}\right|= \pm\left\langle c_{1}, c_{2}\right\rangle$ yields $c_{2}= \pm c_{1}$. So, we get $f(s)=c_{1}(s \pm 1)$ which shows that $f(s)$ is contained in a line through the origin.

If $\kappa$ is a nonzero constant, then $f$ is an open part of a circle with radius $r=\kappa^{-1}$. Thus, we may put $f(s)=a+r e^{i \kappa s}$, where $a$ is a real number. Hence, we get $F(s)=a^{2}+r^{2}+2 a r \cos (\kappa s)$. Substituting this into (6.6) or (6.7) of Lemma 6.2 gives

$$
(\alpha-2 a \kappa \sin (\kappa s))\left(a^{2}+r^{2}+2 a r \cos (\kappa s)\right) \pm a \sin (\kappa s)(r+a \cos (\kappa s))=0,
$$

which implies that $\alpha=a=0$. Hence, the Legendre curve $z$ has constant curvature in $S^{3}(1)$ and $f=r e^{i \kappa s}$. So, $f$ is an open portion of a circle centered at the origin.

The converse is easy to verify.

Remark 6.6. For the special case that $z$ is a closed Legendre curve in $S^{3}(1) \subset \mathbf{C}^{2}$ and $L_{f z}$ is a cone over $z$ (i.e., $f(s)$ is contained in a line through the origin of $\mathbf{C}$ ), Corollary 6.1 was obtained in [27, Theorem 7.1].

\section{Intrinsic properties}

The following result classifies Lagrangian surfaces of constant curvature in $\mathbf{C}^{2}$ which are obtained from our construction method.

THEOREM 7.1. Let $f: I_{1} \rightarrow \mathbf{C}^{*}$ be a unit speed curve in $\mathbf{C}^{*}, p: I_{2} \rightarrow \mathbf{C}$ a curve in $\mathbf{C}$ which does not intersects the curve $f$, and $z: I_{2} \rightarrow S^{3}(1) \subset \mathbf{C}^{2}$ a unit speed Legendre curve. Then the Lagrangian surface $L_{f p z}:\left(I_{1} \times I_{2}, g_{f p z}\right) \rightarrow \mathbf{C}^{2}$ is of constant curvature $K$ if and only if, up to translations on $s$, one of the following eight cases occurs:

(a) $K=0, p$ is a complex number, and $f(s)=p+r e^{i s / r}$, i.e., $f$ is an open portion of circle of radius $r$ centered at $p$;

(b) $K=0, p$ is a real-valued function and, up to rigid motions on $\mathbf{C}, f$ is given by $f(s)=s$;

(c) $K=0, p$ is a complex number and, up to rigid motions on $\mathbf{C}, f$ is given by $f(s)=p+\left(r / \sqrt{1+r^{2}}\right) s^{1+i / r}$ for some nonzero real number $r$

(d) $K=c^{2}, c>0, p(t)=q-e^{-2 i B(t)} / 2 c$ for some $q \in \mathbf{C}$ and some real-valued function $B(t)$ and, up to translations on $s, f$ is given by $f(s)=q+e^{2 i c s} / 2 c$;

(e) $K=c^{2}, c>0, p=c_{0}-a$ with $c_{0} \in \mathbf{C}^{*}$ and, up to translations on $s, f(s)$ is given by 


$$
f=c_{0}-a+a \cos (c s) \exp \left( \pm \frac{i}{a} \int_{0}^{s} \sqrt{1-\varepsilon e^{-4 c^{2} b} \tan ^{2}(c s)} d s\right),
$$

where $\varepsilon=1$ or -1 and $a$ is a real number satisfying $a c=\sqrt{1+\varepsilon e^{-4 c^{2} b}}$ for some real number $b$;

(f) $K=-c^{2}, c>0, p$ is a complex number and, up to translations on $s$,

$$
f(s)=p+a \exp \left(c s+i r \pm i\left(\frac{\sqrt{e^{-2 c s}-c^{2} a^{2}}}{a c}+\sin ^{-1}\left(a c e^{c s}\right)\right)\right),
$$

where $r$ is a real number and $a$ is a positive number;

(g) $K=-c^{2}, c>0, p$ is a complex number and, up to translations on $s$,

$$
f=p+a \sinh (c s) \exp \left(i r \pm \frac{i}{a} \int_{0}^{s} \sqrt{1-a^{2} c^{2} \cosh ^{2}(c s)} \operatorname{csch}(c s) d s\right),
$$

where $a$ and $r$ are real numbers satisfying $0<a^{2}<1 / c^{2}$;

(h) $K=-c^{2}, c>0, p$ is a complex number and, up to translations on $s$,

$$
f=p+a \cosh (c s) \exp \left(i r \pm \frac{i}{a} \int_{0}^{s} \sqrt{1-a^{2} c^{2} \sinh ^{2}(c s)} \operatorname{sech}(c s) d s\right)
$$

where $r$ is a real number and $a$ is a positive number.

Proof. Suppose that $f: I_{1} \rightarrow \mathbf{C}^{*}$ is a unit speed curve, $p(t)$ is a complexvalued function defined on $I_{2}$ which does not intersect $f$, and $z: I_{2} \rightarrow S^{3}(1) \subset \mathbf{C}^{2}$ is a unit speed Legendre curve. Then the induced metric $g_{f p z}$ via $L_{f p z}$ is given by

$$
g_{f p z}=d s^{2}+|f(s)-p(t)|^{2} d t^{2} .
$$

Thus, the Gauss curvature $K$ of $L_{f p z}$ satisfies

$$
G_{s s}=-K G, \quad G=|f(s)-p(t)| .
$$

Case (1). $\quad K=0 . \quad$ Solving (7.1) gives

$$
|f(s)-p(t)|^{2}=(A(t) s+B(t))^{2}
$$

for some real-valued functions $A(t)$ and $B(t)$.

CAse (1.1). $\quad A(t)=0 . \quad$ We obtain from (7.2) that

$$
\left\langle f^{\prime}(s), f(s)-p(t)\right\rangle=0,
$$

which implies

$$
\left\langle f^{\prime}(s), p^{\prime}(t)\right\rangle=\left\langle f^{\prime \prime}(s), p^{\prime}(t)\right\rangle=0 .
$$

If $f^{\prime \prime}=0$, then we have $f(s)=a s+b$ for some complex numbers $a$, $b$ with $|a|=1$. Substituting this into (7.3) gives $\langle a, p(t)\rangle=\langle a, a s+b\rangle=s+\langle a, b\rangle$ which is impossible. Thus, we get $f^{\prime \prime}(s) \neq 0$. Since $\left|f^{\prime}(s)\right|=1$, we find $\left\langle f^{\prime}, f^{\prime \prime}\right\rangle=0$. Hence, $f^{\prime \prime} \neq 0$ and (7.4) imply that $p$ is constant. So, (7.2) 
shows that $B$ is a positive real number, say $r$. Therefore, by applying a suitable translation on $s$, we have $f(s)=p+r e^{i s / r}, p \in \mathbf{C}$. This gives Case (a).

CASE (1.2). $\quad A(t) \neq 0$. We obtain from (7.2) and $\left|f^{\prime}\right|=1$ that

$$
\begin{aligned}
& \left\langle f^{\prime}(s), f(s)-p(t)\right\rangle=A^{2}(t) s+A(t) B(t), \\
& \left\langle f^{\prime \prime}(s), f(s)-p(t)\right\rangle=A^{2}(t)-1 .
\end{aligned}
$$

CASE (1.2.a). $\quad f^{\prime \prime}=0$. We have $f(s)=a s+c_{1}$ for some complex numbers $a, c_{1}$ with $a=e^{i \psi}, \psi \in \mathbf{R}$. Thus, after applying suitable rotation and translation on $\mathbf{C}$, we get $f(s)=s$. Also, from (7.6) we find $A^{2}=1$. Without loss of generality, we may assume that $A=1$. Substituting these into (7.2) shows that $p(t)$ is a real-valued function given by $p(t)=-B(t)$. This gives Case (b).

CASE (1.2.b). $\quad f^{\prime \prime} \neq 0 . \quad$ Since $f^{\prime \prime}(s)=i \kappa f^{\prime}$ with $\kappa \neq 0$, we obtain from (7.5) and (7.6) that

$$
f(s)=p(t)+\left(A^{2}(t) s+A(t) B(t)\right) f^{\prime}(s)+\left(\frac{A^{2}(t)-1}{\kappa(s)}\right) i f^{\prime}(s) .
$$

By differentiating (7.7) with respect to $s$, we find $(A s+B) A \kappa^{3}=\left(A^{2}-1\right) \kappa^{\prime}$, which is nothing but

$$
\left(\frac{1}{\kappa^{2}}\right)^{\prime}=\frac{2 A^{2}(t) s}{1-A^{2}(t)}+\frac{2 A(t) B(t)}{1-A^{2}(t)} .
$$

Thus we get

$$
\left(\frac{1}{\kappa^{2}}\right)^{\prime \prime}=\frac{2 A^{2}(t)}{1-A^{2}(t)}
$$

Since the left-hand-side of (7.9) is independent of $t$, (7.9) implies that there exists a nonzero real number $a$ such that

$$
\left(\frac{1}{\kappa^{2}}\right)^{\prime \prime}=2 a, \quad A^{2}(t)=a\left(1-A^{2}(t)\right) .
$$

From these we obtain $1 / \kappa^{2}=a s^{2}+c_{1} s+c_{2}$ for some real numbers $c_{1}, c_{2}$. So, by applying a suitable translation on $s$, we have

$$
\frac{1}{\kappa^{2}}=a s^{2}+b, \quad A^{2}=\frac{a}{a+1}
$$

for some real number $b$. From (7.8) and (7.10), we find $B=0$. Hence, (7.7) reduces to

$$
f(s)=p+\left(\frac{a s}{a+1}-\frac{i}{(a+1) \kappa(s)}\right) f^{\prime}(s)
$$

which implies that $p$ is constant. 
CONSTRUCTION OF LAGRANGIAN SURFACES IN COMPLEX EUCLIDEAN PLANE 103

CASE (1.2.b.i). $\quad b=0$. In this case, we get $a>0$. Let us put $a=r^{2}$ with $r>0$, so we find from (7.10) that $\kappa=1 /(r s)$. Substituting this into (7.11) gives

$$
f(s)=p+\frac{r(r-i) s}{r^{2}+1} f^{\prime}(s) .
$$

After solving (7.12) we have $f(s)=p+\mu s^{1+i / r}$ for some complex numbers $\mu$. Since $\left|f^{\prime}\right|=1$, we find $|\mu|=r / \sqrt{1+r^{2}}$. Therefore, after applying a suitable rigid motion on $\mathbf{C}$, we obtain

$$
f(s)=p+\frac{r s^{1+i / r}}{\sqrt{1+r^{2}}} .
$$

This gives Case (c).

CASE (1.2.b.ii). $\quad b \neq 0 . \quad$ We obtain from (7.10) and (7.11) that

$$
f^{\prime}(s)=\frac{(a+1)\left(a s \pm i \sqrt{a s^{2}+b}\right)}{a(a+1) s^{2}+b}(f(s)-p)
$$

which implies

$$
|f-p|^{2}=\frac{a(1+a) s^{2}+b}{(1+a)^{2}} .
$$

Thus, by comparing (7.2) and (7.15), we conclude that $b=0$ which is a contradiction. Hence this case is impossible.

Case (2). $K=c^{2}, c>0 . \quad$ Solving (7.1) gives

$$
|f(s)-p(t)|=A(t) \cos (c s+B(t))
$$

for some real-valued functions $A(t) \neq 0$ and $B(t)$. Thus we have

$$
\begin{aligned}
\left\langle f^{\prime}(s), f(s)-p(t)\right\rangle & =-\frac{c}{2} A^{2} \sin (2 c s+2 B), \\
\left\langle f^{\prime \prime}(s), f(s)-p(t)\right\rangle & =-c^{2} A^{2} \cos (2 c s+2 B)-1 .
\end{aligned}
$$

If $f^{\prime \prime}=0$, then (7.18) gives $c^{2} A^{2}(t) \cos (2 c s+2 B(t))+1=0$ for all $t$, which is impossible. Thus, $f^{\prime \prime} \neq 0$, and $f^{\prime}(s), f^{\prime \prime}(s) / \kappa$ form an orthonormal frame. Hence, using (7.17) and (7.18), we have

$$
f(s)=p(t)-\left\{\frac{c}{2} A^{2} \sin (2 c s+2 B)+\frac{i}{\kappa}\left(c^{2} A^{2} \cos (2 c s+2 B)+1\right)\right\} f^{\prime}(s) .
$$

Differentiating (7.19) with respect to $s$ gives

$$
\kappa^{\prime}(s)\left(1+c^{2} A^{2}(t) \cos (2 c s+2 B(t))\right)=\frac{c}{2}\left(\kappa^{3}-4 c^{2} \kappa\right) A^{2}(t) \sin (2 c s+2 B(t)) .
$$


CASE (2.a). $\quad \kappa$ is constant. In this case, (7.20) implies that $\kappa^{2}=4 c^{2}$. Hence, $f$ is contained in a circle of radius $1 / 2 c$. Without loss of generality, we may put $\kappa=2 c$. Since $f(s)$ is a unit speed curve, we may also put

$$
f(s)=q+\frac{e^{2 i c s}}{2 c}
$$

for some $q \in \mathbf{C}$. Substituting this into (7.17) and (7.18) gives

$$
\begin{aligned}
\left\langle i e^{2 i c s}, q-p(t)\right\rangle & =-\frac{c}{2} A^{2} \sin (2 c s+2 B), \\
2\left\langle e^{2 i c s}, q-p(t)\right\rangle & =c A^{2} \cos (2 c s+2 B) .
\end{aligned}
$$

From these two equations we get

$$
2 q=2 p(t)+c A^{2}(t) e^{-2 i B} .
$$

Combining this with $(7.21)$ yields

$$
f(s)=p(t)+\frac{c}{2} A^{2}(t) e^{-2 i B}+\frac{e^{2 i c s}}{2 c}
$$

Thus, we find

$$
4 c^{2}|f(s)-p|^{2}=\left(c^{2} A^{2}-1\right)^{2}+4 c^{2} A^{2} \cos ^{2}(c s+B) .
$$

Comparing (7.26) with (7.16) gives $A=1 / c$. Hence, (7.25) reduces to

$$
f(s)=p(t)+\frac{e^{-2 i B}+e^{2 i c s}}{2 c} .
$$

CASE (2.a.i). $\quad B$ is constant. If we put $B=b$, then (7.27) implies that $p$ is constant and

$$
f(s)=p+\frac{e^{-2 i b}+e^{2 i c s}}{2 c},
$$

which gives Case (d) with constant $B$.

CASE (2.a.ii). $\quad B$ is non-constant. By applying $A=1 / c$, we obtain from (7.21) and (7.24) that

$$
f(s)=q+\frac{e^{2 i c s}}{2 c}, \quad p(t)=q-\frac{e^{-2 i B(t)}}{2 c},
$$

where $q \in \mathbf{C}$ and $B(t)$ is an arbitrary real-valued function. This gives Case (d) with non-constant $B$.

CASE (2.b). $\quad \kappa$ is non-constant. Solving (7.20) yields

$$
\kappa(s)= \pm \frac{2 c\left(1+c^{2} A^{2} \cos (2 c s+2 B)\right)}{\sqrt{\left(1+c^{2} A^{2} \cos (2 c s+2 B)\right)^{2} \mp e^{-8 c^{2} b}}}
$$


for some real number $b$. Since $\kappa$ is non-constant, we see from (7.30) that $A \neq 0$ and $B$ is constant. Without loss of generality, we may assume that $B=0$.

Substituting (7.30) into (7.19) gives

$$
f=p-\left[\frac{c}{2} A^{2} \sin (2 c s) \pm \frac{i}{2 c} \sqrt{\left(1+c^{2} A^{2} \cos (2 c s)\right)^{2} \mp e^{-8 c^{2} b}}\right] f^{\prime},
$$

which implies that

$$
4 c^{2}|f-p|^{2}=4 c^{2} A^{2} \cos ^{2}(c s)+\left(1-c^{2} A^{2}\right)^{2} \mp e^{-8 c^{2} b} .
$$

By comparing (7.32) with (7.16), we get

$$
A^{2}(t)=\left(1+\varepsilon e^{-4 c^{2} b}\right) / c^{2}, \quad \varepsilon=1 \text { or }-1,
$$

which implies that $A$ is a real number, say $a$, satisfying $a^{2} c^{2}=1+\varepsilon e^{-4 c^{2} b}$. We may assume that $a=\sqrt{1+\varepsilon e^{-4 c^{2} b}} / c$. Thus, (7.31) can be written as

$$
f^{\prime}=-2 c\left\{\frac{a^{2} c^{2} \sin (2 c s) \mp i \sqrt{\left(1+a^{2} c^{2} \cos (2 c s)\right)^{2}-e^{-8 c^{2} b}}}{1+a^{4} c^{4}+2 a^{2} c^{2} \cos (2 c s)-e^{-8 c^{2} b}}\right\}(f-p) .
$$

So, by applying $a^{2} c^{2}=1+\varepsilon e^{-4 c^{2} b}$, we have

$$
f^{\prime}=-\left(c \tan (c s) \mp \frac{i}{a} \sqrt{1-\varepsilon e^{-4 c^{2} b} \tan ^{2}(c s)}\right)(f-p) .
$$

Solving (7.33) gives

$$
f=p+\alpha \cos (c s) \exp \left( \pm \frac{i}{a} \int_{s_{0}}^{s} \sqrt{1-\varepsilon e^{-4 c^{2} b} \tan ^{2}(c s)} d s\right)
$$

for some $s_{0}, \alpha \in \mathbf{R}$ with $a=\sqrt{1+\varepsilon e^{-4 c^{2} b}} / c$. Since $|f-p|^{2}=a^{2} \cos ^{2}(c s)$, we must have $\alpha= \pm a$. Without loss of generality, we may assume that $\alpha=a$. Moreover, we may also assume that $s_{0}=0$ by applying a suitable translation on $s$ if necessary. Thus, we have

$$
f=p+a \cos (c s) \exp \left( \pm \frac{i}{a} \int_{0}^{s} \sqrt{1-\varepsilon e^{-4 c^{2} b} \tan ^{2}(c s)} d s\right),
$$

which implies that $p=c_{0}-a, c_{0}=f(0) \in \mathbf{C}^{*}$. Consequently, we obtain

$$
f=c_{0}-a+a \cos (c s) \exp \left( \pm \frac{i}{a} \int_{0}^{s} \sqrt{1-\varepsilon e^{-4 c^{2} b} \tan ^{2}(c s)} d s\right),
$$

whose curvature function is given by

$$
\kappa= \pm \frac{2 a^{2} c^{2}-e^{-4 c^{2} b} \sec ^{2}(c s)}{a \sqrt{1-\varepsilon e^{-4 c^{2} b} \tan ^{2}(c s)}} .
$$

This gives Case (e).

CASE (3). $K=-c^{2}, c>0$. Solving (7.1) gives 


$$
|f(s)-p(t)|=\alpha(t) \cosh (c s)+\beta(t) \sinh (c s)
$$

for some real-valued functions $\alpha(t)$ and $\beta(t)$. We divide this into several cases.

CAse (3.1). $\alpha= \pm \beta$. In this case, (7.36) becomes

$$
|f(s)-p(t)|=a(t) e^{ \pm c s},
$$

where $a$ is a positive function. Without loss of generality, we may assume that

$$
|f(s)-p(t)|=a(t) e^{c s} .
$$

Thus we have

$$
\begin{aligned}
& \left\langle f^{\prime}(s), f(s)-p(t)\right\rangle=c a^{2} e^{2 c s}, \\
& \left\langle f^{\prime \prime}(s), f(s)-p(t)\right\rangle=2 c^{2} a^{2} e^{2 c s}-1 .
\end{aligned}
$$

If $f^{\prime \prime}=0$, then (7.39) gives $2 c^{2} a^{2}(t) e^{2 c s}=1$ for all $t$ which is impossible. Thus, we have $f^{\prime \prime} \neq 0$. So, by applying (7.38) and (7.39), we find

$$
f(s)=p(t)+\left\{c a^{2} e^{2 c s}+\frac{i}{\kappa}\left(2 c^{2} a^{2} e^{2 c s}-1\right)\right\} f^{\prime}(s) .
$$

Differentiating (7.40) with respect to $s$ yields

$$
\kappa^{\prime}(s)+a^{2}(t) c\left(4 c^{2} \kappa(s)+\kappa^{3}(s)-2 c \kappa^{\prime}(s)\right) e^{2 c s}=0 .
$$

From this we know that $\kappa^{\prime} \neq 0$ and $a$ is positive. In views of (7.37), we may put

$$
f(s)=p(t)+a e^{c s+i \theta},
$$

for some real-valued function $\theta=\theta(s, t)$. By applying (7.41) and $\left|f^{\prime}(s)\right|=1$, we find

$$
\theta_{s}= \pm \sqrt{e^{-2 c s}-c^{2} a^{2}} / a
$$

Hence, $\theta$ is given by

$$
\theta=\mp\left(\frac{\sqrt{e^{-2 c s}-c^{2} a^{2}}}{a c}+\sin ^{-1}\left(a c e^{c s}\right)\right)+\psi(t)
$$

for some real-valued function $\psi=\psi(t)$. Let us put

$$
\varphi(s)=\mp\left(\frac{\sqrt{e^{-2 c s}-c^{2} a^{2}}}{\alpha c}+\sin ^{-1}\left(a c e^{c s}\right)\right) .
$$

Then we obtain from (7.42) that $\varphi(s)=\theta-\psi(t)$. Now, by differentiating (7.41) with respect to $t$ and using (7.42), we find

$$
0=p^{\prime}(t)+i a \psi^{\prime}(t) e^{c s} e^{i \varphi(s)} e^{i \psi(t)}
$$

for all $t$ which is impossible unless $p$ and $\psi$ are constant. Thus, up to translations on $s$, we have 
CONSTRUCTION OF LAGRANGIAN SURFACES IN COMPLEX EUCLIDEAN PLANE 107

$$
f(s)=p+a e^{c s+i \varphi(s)+i \psi},
$$

where $p$ is a complex number and $\psi$ is a real number, say $r$. Thus, we obtain

$$
f(s)=p+a \exp \left(c s \mp i\left(\frac{\sqrt{e^{-2 c s}-c^{2} a^{2}}}{a c}+\sin ^{-1}\left(a c e^{c s}\right)\right)+i r\right) .
$$

This gives Case (f).

CASE (3.2). $\alpha(t)=0 . \quad$ In this case, (7.36) reduces to

$$
|f(s)-p(t)|=\beta(t) \sinh (c s) \text {. }
$$

Thus we have

$$
\begin{aligned}
\left\langle f^{\prime}(s), f(s)-p(t)\right\rangle & =\frac{c}{2} \beta^{2}(t) \sinh (2 c s), \\
\left\langle f^{\prime \prime}(s), f(s)-p(t)\right\rangle & =c^{2} \beta^{2}(t) \cosh (2 c s)-1 .
\end{aligned}
$$

If $f^{\prime \prime}=0$, then (7.48) gives $c^{2} \beta^{2}(t) \cosh (2 c s)=1$ for all $t$ which is impossible. So, we have $f^{\prime \prime}=i \kappa f^{\prime} \neq 0$. Hence, (7.47) and (7.48) imply that

$$
f(s)=p(t)+\left\{\frac{c}{2} \beta^{2} \sinh (2 c s)+\frac{i}{\kappa}\left(c^{2} \beta^{2} \cosh (2 c s)-1\right)\right\} f^{\prime}(s) .
$$

Differentiating (7.49) with respect to $s$ gives

$$
2 \kappa^{\prime}(s)\left(1-c^{2} \beta^{2}(t) \cosh (2 c s)\right)+c \kappa(s) \beta^{2}(t)\left(4 c^{2}+\kappa^{2}\right) \sinh (2 c s)=0
$$

which implies that $\beta$ is a nonzero real number, say $a$. Without loss of generality, we may assume $a>0$. Thus, in views of (7.46), we may put

$$
f(s)=p(t)+a \sinh (c s) e^{i \theta}
$$

for some real-valued function $\theta=\theta(s, t)$. From (7.50) and $\left|f^{\prime}(s)\right|=1$, we find

$$
a^{2} \sinh ^{2}(c s) \theta_{s}^{2}=1-a^{2} c^{2} \cosh ^{2}(c s),
$$

which implies that $a^{2} c^{2} \leq 1$ due to $\cosh (c s) \geq 1$. If $a^{2} c^{2}=1$, then (7.51) becomes

$$
\left(1+a^{2} \theta_{s}^{2}\right) \sinh ^{2}(c s)=0
$$

for all $s$ which is impossible. So, we must have $a^{2}<c^{-2}$. Hence, we obtain from (7.51) that

$$
\theta_{s}= \pm \frac{\sqrt{1-a^{2} c^{2} \cosh ^{2}(c s)}}{a \sinh (c s)}
$$

If we put

$$
\varphi(s)= \pm \int_{0}^{s} \frac{\sqrt{1-a^{2} c^{2} \cosh ^{2}(c s)}}{a \sinh (c s)} d s
$$


then we obtain from (7.52) that $\theta(s, t)=\varphi(s)+\psi(t)$ for some real-valued function $\psi(t)$. Substituting this into (7.50) gives

$$
f(s)=p(t)+a \sinh (c s) \exp \left( \pm i \int_{0}^{s} \frac{\sqrt{1-a^{2} c^{2} \cosh ^{2}(c s)}}{a \sinh (c s)} d s+i \psi(t)\right)
$$

By differentiating (7.55) with respect to $t$, we get

$$
i p^{\prime}(t) e^{-i \psi(t)}=a \sinh (c s) e^{i \varphi(s)} \psi^{\prime}(t),
$$

which is impossible unless both $p$ and $\psi$ are constant. Therefore, up to translations on $s$, we have

$$
f(s)=p+a \sinh (c s) \exp \left(i r \pm \frac{i}{a} \int_{0}^{s} \sqrt{1-a^{2} c^{2} \cosh ^{2}(c s)} \operatorname{csch}(c s) d s\right)
$$

where $p$ is a complex number, $a$ is a nonzero real number and $r$ is a real number. Consequently, we obtain Case (g).

CASE (3.3). $\alpha^{2}>\beta^{2}$. We put $A(t)=\sqrt{\alpha^{2}-\beta^{2}}, \alpha=A \cosh \eta$ and $\beta=$ $A \sinh \eta$. Then (7.36) becomes

$$
|f(s)-p(t)|=A(t) \cosh (c s+\eta(t)),
$$

which implies that

$$
\begin{aligned}
& \left\langle f^{\prime}(s), f(s)-p(t)\right\rangle=\frac{c}{2} A^{2}(t) \sinh (2 c s+2 \eta), \\
& \left\langle f^{\prime \prime}(s), f(s)-p(t)\right\rangle=c^{2} A^{2}(t) \cosh (2 c s+2 \eta)-1 .
\end{aligned}
$$

Equation (7.58) shows that $f^{\prime \prime}=i \kappa f^{\prime} \neq 0$. So, we find from (7.57) and (7.58) that

$$
f(s)=p(t)+\left\{\frac{c}{2} A^{2}(t) \sinh (2 c s+2 \eta)+\frac{i}{\kappa}\left(c^{2} A^{2} \cosh (2 c s+2 \eta)-1\right)\right\} f^{\prime}(s) .
$$

Differentiating this equation with respect to $s$ gives

$$
2 \kappa^{\prime}(s)\left(1-c^{2} A^{2}(t) \cosh (2 c s+2 \eta)\right)+c \kappa(s) A^{2}(t)\left(4 c^{2}+\kappa^{2}\right) \sinh (2 c s+2 \eta)=0
$$

which can be written as

$$
A^{2}(t)=\frac{2 \kappa^{\prime}(s)}{2 c^{2} \kappa^{\prime} \cosh (2 c s+2 \eta)-c \kappa\left(4 c^{2}+\kappa^{2}\right) \sinh (2 c s+2 \eta)} .
$$

By differentiating (7.59) with respect $s$ gives

$$
\operatorname{coth}(2 c s+2 \eta)=\frac{\left(4 c^{2}+\kappa^{2}\right) \kappa^{\prime \prime}-3 \kappa \kappa^{\prime 2}}{2 c\left(4 c^{2}+\kappa^{2}\right) \kappa^{\prime}(s)} .
$$

Since the right-hand-side of (7.60) depends only on $s$, (7.60) implies that $\eta(t)$ is 
CONSTRUCTION OF LAGRANGIAN SURFACES IN COMPLEX EUCLIDEAN PLANE 109

constant which is a real number. Thus, by applying (7.59) we know that $A$ is also constant which is a positive number.

Let us denote $\eta$ and $A$ by $r$ and $a$, respectively. Then, by using (7.56), we have

$$
f(s)=p(t)+a \cosh (c s+r) e^{i \theta}
$$

for some real-valued function $\theta=\theta(s, t)$. So, by applying a suitable translation on $s$, we have

$$
f(s)=p(t)+a \cosh (c s) e^{i \theta}
$$

for some real-valued function $\theta=\theta(s, t)$.

From (7.61) and $\left|f^{\prime}(s)\right|=1$, we obtain

$$
a^{2} \theta_{s}^{2} \cosh ^{2}(c s)=1-a^{2} c^{2} \sinh ^{2}(c s) .
$$

Thus, we obtain

$$
\theta_{s}= \pm \frac{\sqrt{1-a^{2} c^{2} \sinh ^{2}(c s)}}{a \cosh (c s)}
$$

Hence, we get

$$
\theta=\phi(s)+\psi(t), \quad \phi(s)= \pm \int_{0}^{s} \frac{\sqrt{1-a^{2} c^{2} \sinh ^{2}(c s)}}{a \cosh (c s)} d s
$$

where $\psi(t)$ is a real-valued function. By differentiating (7.61) with respect to $t$ and applying (7.62), we find

$$
i p^{\prime}(t)=a \psi^{\prime}(t) \cosh (c s) e^{i \phi(s)} e^{i \psi(t)},
$$

which is impossible unless $p$ and $\psi$ are both constant. Hence, we have

$$
f(s)=p+a \cosh (c s) e^{i \phi(s)} e^{i \psi}
$$

where $a>0, \psi$ are real number and $p$ is a complex number. This gives Case (h).

CASE (3.4). $\quad \alpha^{2}<\beta^{2}$. We put

$$
A(t)=\sqrt{\beta^{2}-\alpha^{2}}, \quad \alpha=A \sinh \eta, \quad \beta=A \cosh \eta,
$$

then we get from (7.36) that

$$
|f(s)-p(t)|=A(t) \sinh (c s+\eta(t)) .
$$

From (7.63) we find

$$
\begin{aligned}
\left\langle f^{\prime}(s), f(s)-p(t)\right\rangle & =\frac{c}{2} A^{2}(t) \sinh (2 c s+2 \eta), \\
\left\langle f^{\prime \prime}(s), f(s)-p(t)\right\rangle & =c^{2} A^{2}(t) \cosh (2 c s+2 \eta)-1 .
\end{aligned}
$$


Thus, we may applying the same argument as Case (3.3) to conclude that $A$ and $\eta$ are real numbers, say $a$ and $\gamma$, respectively. So, after applying a suitable translation on $s$, we obtain $|f(s)-p(t)|=a \sinh (c s)$. Thus, this reduces to Case (3.2).

Conversely, by straight-forward computation, we know that all of the associated Lagrangian surfaces obtained from Cases (a)-(i) are of constant curvature.

Remark 7.1. Up to rigid motions on $\mathbf{C}^{2}$, the Lagrangian surfaces of constant curvature obtained in Theorem 7.1 are given respectively by

$\left(\mathrm{a}^{\prime}\right) L_{f p z}(s, t)=r e^{i s / r} z(t)$;

$\left(\mathrm{b}^{\prime}\right) L_{f p z}(s, t)=s z(t)-\int_{0}^{t} p(t) z^{\prime}(t) d t$

(c') $L_{f p z}(s, t)=\frac{r}{\sqrt{1+r^{2}}} s^{1+i / r} z(t)$;

$\left(\mathrm{d}^{\prime}\right) L_{f p z}(s, t)=\frac{1}{2 c}\left\{z(t) e^{2 i c s}+\int_{0}^{t} e^{-2 i B(t)} z^{\prime}(t) d t\right\}$;

(e') $L_{f p z}(s, t)=a z(t) \cos (c s) \exp \left( \pm \frac{i}{a} \int_{0}^{s} \sqrt{1-\varepsilon e^{-4 c^{2} b} \tan ^{2}(c s)} d s\right)$; with $a=$ $\frac{1}{c} \sqrt{1+\varepsilon e^{-4 c^{2} b}}$ and $\varepsilon=1$ or -1 ;

(f') $L_{f p z}(s, t)=a z(t) \exp \left(c s \pm i\left(\frac{\sqrt{e^{-2 c s}-a^{2} c^{2}}}{a c}+\sin ^{-1}\left(a c e^{c s}\right)\right)\right)$;

$\left(\mathrm{g}^{\prime}\right) L_{f p z}(s, t)=a z(t) \sinh (c s) \exp \left( \pm \frac{i}{a} \int_{0}^{s} \sqrt{1-a^{2} c^{2} \cosh ^{2}(c s)} \operatorname{csch}(c s) d s\right) ;$

$\left(\mathrm{h}^{\prime}\right) L_{f p z}(s, t)=a z(t) \cosh (c s) \exp \left( \pm \frac{i}{a} \int_{0}^{s} \sqrt{1-a^{2} c^{2} \sinh ^{2}(c s)} \operatorname{sech}(c s) d s\right)$.

Remark 7.2. For each closed Legendre curve $z=z(t)$ in $S^{3}(1)$, the Lagrangian surface defined by $\left(a^{\prime}\right)$ is complete. Such a Lagrangian surface is a flat torus which is contained in $S^{3}(r)$.

Remark 7.3. The local classification of Lagrangian surfaces of constant curvature in the complex Euclidean plane have been obtained [11]. And the local classification of Lagrangian surfaces of constant curvature in the complex projective plane or in the complex hyperbolic plane was established in a series of recent articles $[12,13,14,15,16]$.

Acknowledgement. The author thanks the referee for the many valuable suggestions and very careful reading of the manuscript.

\section{REFERENCES}

[1] R. Airama, Lagrangian surfaces in the complex 2-space, Proceedings of the Fifth International Workshop on Differential Geometry (Taegu, 2000), Kyungpook Natl. Univ., Taegu, 2001, 25-29. 
CONSTRUCTION OF LAGRANGIAN SURFACES IN COMPLEX EUCLIDEAN PLANE 111

[2] R. Aiyama, Totally real surfaces in the complex 2-space, Steps in differential geometry (Debrecen, 2000), Debrecen, 2001, 15-22.

[3] R. Airama, Lagrangian surfaces with circle symmetry in the complex 2-space, Michigan Math. J. 52 (2004), 491-506.

[4] I. Castro, The Lagrangian version of a theorem of J. B. Meusnier, Summer School on Differential Geometry (Coimbra, 1999), Univ. Coimbra, Coimbra, 1999, 83-89.

[5] I. Castro and F. Urbano, On a minimal Lagrangian submanifold of $\mathbf{C}^{n}$ foliated by spheres, Michigan Math. J. 46 (1998), 71-82.

[ 6 ] I. Castro and F. Urbano, Examples of unstable Hamiltonian-minimal Lagrangian tori in $\mathbf{C}^{2}$, Compositio Math. 111 (1998), 1-14.

[7] B.-Y. CHEN, Complex extensors and Lagrangian submanifolds in complex Euclidean spaces, Tohoku Math. J. 49 (1997), 277-297.

[ 8 ] B.-Y. CHEN, Interaction of Legendre curves and Lagrangian submanifolds, Israel J. Math. 99 (1997), 69-108.

[ 9 ] B.-Y. CHEN, Representation of flat Lagrangian $H$-umbilical submanifolds in complex Euclidean spaces, Tohoku Math. J. 51 (1999), 13-20.

[10] B.-Y. CHEN, Lagrangian surfaces of constant curvature in complex Euclidean plane, Tohoku Math J. 56 (2004), 289-298.

[11] B.-Y. CHEN, Classification of Lagrangian surfaces of constant curvature in complex Euclidean plane, Proc. Edinburgh Math. Soc. 48 (2005), 337-364.

[12] B.-Y. CHEN, Maslovian Lagrangian surfaces of constant curvature in complex projective or complex hyperbolic planes, Math. Nachr. 278 (2005), 1242-1281.

[13] B.-Y. CHEN, Classification of Lagrangian surfaces of constant curvature in complex complex projective planes, J. Geom. Phys. 53 (2005), 428-460.

[14] B.-Y. CHEN, Classification of Lagrangian surfaces of constant curvature in complex hyperbolic planes, J. Geom. Phys. 55 (2005), 399-439.

[15] B.-Y. CHEN, Three additional families of Lagrangian surfaces of constant curvature in complex projective plane, J. Geom. Phys. 56 (2006), 666-669.

[16] B.-Y. CHEN, Classification of Lagrangian surfaces of constant curvature in complex hyperbolic planes, II, Soochow J. Math. 32 (2006).

[17] B.-Y. Chen, F. Dillen, L. Verstraelen and L. Vrancken, Lagrangian isometric immersions of a real-space-form $M^{n}(c)$ into a complex-space-form $\tilde{M}^{n}(4 c)$, Math. Proc. Cambridge Phil. Soc. 124 (1998), 107-125.

[18] B.-Y. CHEN AND O. J. GARAY, Maslovian Lagrangian isometric immersions of real space forms into complex space forms, Japan. J. Math. 30 (2004), 227-281.

[19] B.-Y. Chen And J. M. Morvan, Geometrie des surfaces lagrangiannes de C ${ }^{2}$, J. Math. Pures Appl. 66 (1987), 321-325.

[20] B.-Y. Chen and K. Ogiue, On totally real submanifolds, Trans. Amer. Math. Soc. 193 (1974), 257-266.

[21] R. Harvery and H. B. Lawson, Calibrated geometries, Acta Math. 148 (1982), 47157.

[22] F. Hélein and P. Romon, Hamiltonian stationary Lagrangian surfaces in $\mathbf{C}^{2}$, Comm. Anal. Geom. 10 (2002), 79-126.

[23] D. Joyce, Special Lagrangian $m$-folds in $\mathbf{C}^{m}$ with symmetries, Duke Math. J. 115 (2002), $1-51$.

[24] M. Kriele AND L. VRancKen, Minimal Lagrangian submanifolds of Lorentzian complex space forms with constant sectional curvature, Arch. Math. (Basel) 72 (1999), 223-232.

[25] R. K. Nagle, E. B. SAfF and A. D. Snider, Differential equations and boundary value problems, Addison Wesley, New York, 2004. 
[26] Y.-G. OH, Second variation and stabilities of minimal Lagrangian submanifolds in Kaehler manifolds, Invent. Math. 101 (1990), 501-519.

[27] R. Schoen AND J. Wolfson, Minimizing area among Lagrangian surfaces: the mapping problem, J. Differential Geom. 58 (2001), 1-86.

Department of Mathematics

Michigan State University

EAst LANSING

MichigAN 48824-1027

U.S.A.

E-mail: bychen@math.msu.edu 